\title{
Adenine nucleotide translocase 2: an emerging player in cancer
}

\author{
Seung Hyun Baik ${ }^{1}$ and Jongkuen Lee ${ }^{2 *}$ \\ ${ }^{1}$ Division of Reproductive Science in Medicine, Department of Obstetrics and Gynecology, Feinberg School of Medicine, Northwestern University, Chicago, IL, USA \\ ${ }^{2}$ Department of Oncological Sciences and Tisch Cancer Institute, Icahn School of Medicine at Mount Sinai, New York, NY, USA
}

\begin{abstract}
Adenine nucleotide translocase (ANT) is an integral protein located in the inner mitochondrial membrane and plays an important role in maintaining ATP/ADP ratio, thereby contributing to the energy-supplying function of mitochondria. It also mediates mitochondria-mediated cell death through interactions with Bax and $\mathrm{Bcl}-2$, pro- and anti-apoptotic proteins, respectively. While the overall function of ANT is known, the diverse functions of ANT isoforms have not been investigated in depth. Of the four ANT isoforms (ANT1, ANT2, ANT3, and ANT4), ANT2 has gained many interests due to its aberrant expression in cancers. Here, we discuss the importance of ANT2 in proliferating cells, and highlight its cytoprotective and anti-apoptotic functions in cancer cells. Although the exact tumor-supportive mechanisms of ANT2 remain unclear, accumulating evidence suggests that ANT2 could be a promising target for cancer therapy.
\end{abstract}

\section{Discussion}

Following the observation that cancers prefer glycolytic metabolism to generate energy for cellular processes even in the presence of oxygen (aerobic glycolysis), Warburg proposed mitochondrial respiration defect as the underlying cause of cancer $[1,2]$. However, we now understand that there are many genetic mutations in cancers, altering cellular metabolism without necessarily impairing mitochondrial function. For instance, oncogenic mutations and amplification of K-ras, c-Myc, and phosphatidylinositol-3 kinase (PI3K) or loss of tumor suppressor genes such as p53 and phosphatase and tensin homolog (Pten) promote glycolysis [3]. Since mitochondria are implicated in various biological activities, including apoptosis and inflammation, the role of mitochondria in cancer is not as simple as Warburg had envisioned [4,5]. Studies of mitochondrial function in diseases have alluded to the significance of maintaining both ATP/ADP ratio and the mitochondrial transmembrane electrochemical gradient $(\Delta \Psi \mathrm{m})$ generated by oxidative phosphorylation [6,7]. The maintenance of the ATP/ADP ratios is partly regulated by the adenine nucleotide translocase (ANT), an integral protein located abundantly in the inner mitochondrial membrane. The physiological role of this protein is to catalyze the exchange of ATP/ADP across the mitochondrial inner membrane [8]. By using carboxyatractyloside, an ANT specific inhibitor, one study solved the crystal structure of bovine heart ANT [9]. The crystal analysis revealed six transmembrane helices with a hexapeptide carrying the signature of nucleotide carriers (RRRMMM) at its bottom even though the exact molecular mechanism of ATP/ADP exchange still remains elusive. ANT structure and earlier biochemical findings altogether imply that ANT plays an essential role in cellular energy metabolism by coupling mitochondrial ATP synthesis to cytosolic delivery. In addition, ANT is a crucial component of the mitochondrial permeability transition pore complex (PTPC) and plays a major role in mitochondria-mediated cell death [10]. ANT and members of the Bcl-2 family create contact sites between the mitochondrial inner and outer membranes [11]. ANT cooperates with Bax, a pro-apoptotic protein, within the PTPC to modulate mitochondrial membrane permeabilization (MMP) and mediate apoptosis responses [12] whereas interaction with Bcl-2 exhibits inhibition of the lethal pore formation [13]. Also, viral protein $\mathrm{R}$, an apoptogenic protein encoded by HIV-1, induces MMP via a direct interaction with ANT [14]. However, Kokoszka et al. [15] showed that mitochondria from livers of ANT1/2-deficient mice still triggered MMP and concluded that ANT1/2 are non-essential components of the PTPC, casting doubt on the role of ANT in MMP. Thus, it remains to be further investigated whether ANT is a central component or just one of the regulators of the PTPC.

Human ANT has four isoforms (ANT1, ANT2, ANT3, and ANT4) and their expressions are various, depending on tissue types, developmental stages, and proliferation status [11] (Table 1). ANT1 is highly expressed in non-dividing, terminally differentiated muscle and heart cells [16]. ANT2 is specifically abundant in tissues that are able to proliferate and/or regenerate such as in lymphoid cells, liver, and various cancers [17-19]. ANT3 is ubiquitously expressed at lower levels and ANT4 is essential for spermatogenesis in testis $[16,20]$. This suggests that the expressions of the various ANT genes are contextspecific and tightly regulated. Indeed, the promoter structure and organization of four ANT genes are different and their expressions can be controlled epigenetically [11]. For example, DNA methylation - that results in transcriptional silencing - of ANT4 promoter is observed in somatic cells whereas its hypomethylation is found in pluripotent stem

Correspondence to: Jongkuen Lee, Department of Oncological Sciences and Tisch Cancer Institute, Icahn School of Medicine at Mount Sinai, Hess Center for Science and Medicine, 1470 Madison Avenue, New York, NY 10029, USA, Tel: 212-824-9230; E-mail: jongkuen.lee@mssm.edu

Key words: mitochondria, adenine nucleotide translocase, apoptosis, tumor development

Received: August 25, 2016; Accepted: September 18, 2016; Published: September 21, 2016 
Table 1. Human ANT isoforms are differentially expressed depending on tissue types, developmental stages and proliferation status.

\begin{tabular}{|l|l|l|l|l|}
\hline Protein Isoform & Gene & Location & Apoptosis Induction & Expression \\
\hline ANT1 & SLC25A4 & Chr4 & Pro-apoptotic & $\begin{array}{l}\text { Non-dividing, terminally differentiated cells/tissues; skeletal muscles, } \\
\text { hearts and brains }\end{array}$ \\
\hline ANT2 & SLC25A5 & ChrX & Anti-apoptotic & $\begin{array}{l}\text { Proliferative and regenerative undifferentiated cells/tissues; } \\
\text { lymphocytes, kidneys, livers, and hormone dependent cancers }\end{array}$ \\
\hline ANT3 & SLC25A6 & ChrX & Pro-apoptotic & Ubiquitous expression in all tissues \\
\hline ANT4 & SLC25A31 & Chr4 & Anti-apoptotic & Testes \\
\hline
\end{tabular}

cells and germ cells [21]. Given the various structures and expressions of four ANT isoforms, their possible differential functions in regard to cell fate control have been investigated. Although there is no clear evidence showing that each of the four ANT isoforms interacts differently with various members of Bcl-2 family, it exhibits different apoptotic behaviors [8]. Previous studies identified ANT1 and ANT3 as pro-apoptotic factors by showing that their overexpression triggered caspase- 9 and -3 activation via protein-protein interaction within the PTPC, facilitating mitochondria-dependent apoptosis [22,23]. In contrast, accumulating evidence demonstrates that ANT2 and ANT4 are anti-apoptotic, suggesting the specificities of ANT isoforms for apoptosis induction [11].

While there are four ANT isoforms, ANT2 has gained most attention from many research groups due to its markedly upregulated expression in cancers. ANT2 overexpression has been observed in a wide variety of cancers including stomach, lung, liver, ovary, and breast cancers, suggesting its significance in cancer development [24,25]. Jang et al. have described that the two ANT isoforms-ANT1 and ANT3-are barely detectable in breast cancer (MCF7, MDA-231, and SK-BR-3) and ovarian cancer (SK-OV-3 and SNU8) cells, emphasizing that ANT2 overexpression could be a unique feature at least in breast and ovarian cancer development [24]. The role of ANT2 in cancer development is not restricted to its anti-apoptotic behavior. One study proposed that ANT2 could have cytoprotective impacts in cancer cells by reversely importing ATP into the mitochondrial matrix to compensate for the decreased mitochondrial ATP generation [26]. Furthermore, it has been shown that ANT2 is implicated in the PI3K/AKT pathway in breast and liver cancers $[18,27,28]$. While the overexpression of ANT2 correlated with increased Akt phosphorylation, the level of phosphorylated Akt decreased in response to ANT2 suppression. The PI3K/Akt signaling pathway is important in cancer growth, metabolism, survival and motility, and it is likely the most frequently activated pathway in cancers, affecting $30-50 \%$ of tumors [29-31]. Thus, its association with the PI3K/Akt signaling pathway could be important for cancer development although the exact role of ANT2 in this pathway still remains to be elucidated. In addition, ANT2 is shown to regulate multiple microRNAs (miRNAs), which are negative regulators of gene expression, and deregulation of specific miRNAs is commonly found in various tumors $[18,28]$. Previously, we reported that ANT2-regulated miR-19a and miR-96 enhanced the proliferation of human liver cancer cells, and the knockdown of ANT2 negatively regulated miR-19a and miR-96, thereby suppressing tumor growth [18]. Also, one study showed the metabolic role of ANT2 in obesity [32]. The authors demonstrated that saturated fatty acids stimulate adipocyte mitochondrial ANT2, elevating $\mathrm{O}_{2}$ consumption. Increased adipocyte $\mathrm{O}_{2}$ consumption leads to inflammation and insulin resistance in obesity by inducing hypoxia. Since a great deal of research shows a significant association with obesity for several cancers, its involvement in insulin sensitivity and glucose tolerance could be relevant to cancer. Altogether, numerous studies suggest that ANT2 is a key player in tumor development although the tumor-supportive mechanisms of
ANT2 are still poorly understood.

As cancer cells depend on the high rate of glycolysis and ANT2 is involved in the glycolytic metabolism, it is suggested as an anti-cancer therapeutic target. Indeed, many studies demonstrate that suppression of ANT2 by shRNA successfully kills cancer cells [17-19]. However, gene therapy using RNA interference has been hindered owing to the inefficiency of delivery in vivo. To solve this problem, a study by Park et al. suggests a method of ANT2 shRNA gene delivery with ultrasound [33]. This combination has led to significant tumor regression upon knockdown of ANT2 in vivo without toxicity, and therefore it provides evidence that ANT2 could be a promising target for cancer therapy.

\section{Conclusion and future directions}

ANT functions as the ATP/ADP carrier that facilitates the exchange of mitochondrial ATP for ADP from the intermembrane space, thus influencing cellular metabolism. Recent studies indicate that ANT isoforms have different effects on cell fate decisions; ANT1 and ANT3 are pro-apoptotic and ANT2 and ANT4 are anti-apoptotic. Given the similarities of sequences between four ANT isoforms, it is surprising that there is functional diversity. One possible explanation is that each isoform has distinct interacting partners or various topological distributions within the mitochondrial inner membrane, regulating MMP in different ways. Thus, a better understanding of the complicated interplay between the ANT isoforms and their partners in diverse situations is required. Moreover, the differences in the gene structures and epigenetic modifications of ANT isoforms may provide a new layer of their specificity for inducing different cellular behaviors. In various cancer types, aberrant expressions of ANT2 are frequently observed. Since cancer cells prefer utilizing the glycolytic pathway, ANT2 could be crucial as ANT2 imports glycolytically generated ATP into the mitochondria, contributing to the maintenance of the mitochondrial membrane potential and prevention of apoptosis. However, the exact role of ANT2 in cancer is not fully understood. Based on the increasing evidence that ANT2 has a positive correlation with tumor progression, ANT2 could be a promising target for cancer therapy. Nevertheless, the fact that normal proliferating cells also express ANT2 should be taken into account and it is required to conduct therapeutic window evaluation to prevent possible adverse effects.

\section{Competing interest statement}

Author has no conflict of interest.

\section{References}

1. Warburg O (1956) On the origin of cancer cells. Science 123: 309-314. [Crossref]

2. Weinhouse S (1956) On respiratory impairment in cancer cells. Science 124: 267-269. [Crossref]

3. Vander Heiden MG, Cantley LC, Thompson CB (2009) Understanding the Warburg effect: the metabolic requirements of cell proliferation. Science 324: 1029-1033. [Crossref] 
4. Zong WX, Rabinowitz JD, White E (2016) Mitochondria and Cancer. Mol Cell 61: 667-676. [Crossref]

5. Giampazolias E, Tait SW (2016) Mitochondria and the hallmarks of cancer. FEBS J 283: 803-814. [Crossref]

6. Klingenberg M (1980) The ADP-ATP translocation in mitochondria, a membrane potential controlled transport. J Membr Biol 56: 97-105. [Crossref]

7. Wallace DC (2001) Mouse models for mitochondrial disease. Am J Med Genet 106 71-93. [Crossref]

8. Chevrollier A, Loiseau D, Reynier P, Stepien G (2011) Adenine nucleotide translocase 2 is a key mitochondrial protein in cancer metabolism. Bba-Bioenergetics 1807: 562-567.

9. Pebay-Peyroula E, Dahout-Gonzalez C, Kahn R, Trézéguet V, Lauquin GJ, et al. (2003) Structure of mitochondrial ADP/ATP carrier in complex with carboxyatractyloside. Nature 426: 39-44. [Crossref]

10. Halestrap AP, Brenner C (2003) The adenine nucleotide translocase: A central component of the mitochondrial permeability transition pore and key player in cell death. Curr Med Chem 10: 1507-1525.

11. Brenner C, Subramaniam K, Pertuiset C, Pervaiz S (2011) Adenine nucleotide translocase family: four isoforms for apoptosis modulation in cancer. Oncogene. 30 : 883-895.

12. Marzo I, Brenner C, Zamzami N, Jurgensmeier JM, Susin SA, et al. (1998) Bax and adenine nucleotide translocator cooperate in the mitochondrial control of apoptosis. Science 281: 2027-2031.

13. Brenner C, Cadiou H, Vieira HL, Zamzami N, Marzo I, et al. (2000) Bcl-2 and Bax regulate the channel activity of the mitochondrial adenine nucleotide translocator. Oncogene 19: 329-336. [Crossref]

14. Jacotot E, Ravagnan L, Loeffler M, Ferri KF, Vieira HLA, et al. (2000) The HIV-1 viral protein $\mathrm{R}$ induces apoptosis via a direct effect on the mitochondrial permeability transition pore. J Exp Med 191: 33-45.

15. Kokoszka JE, Waymire KG, Levy SE, Sligh JE, Cai J, et al. (2004) The ADP/ATP translocator is not essential for the mitochondrial permeability transition pore. Nature 427: 461-465. [Crossref]

16. Doerner A, Pauschinger M, Badorff A, Noutsias M, Giessen S, et al. (1997) Tissuespecific transcription pattern of the adenine nucleotide translocase isoforms in humans. Febs Lett 414: 258-262.

17. Jang JY, Choi Y, Jeon YK, Kim CW (2008) Suppression of adenine nucleotide translocase-2 by vector-based siRNA in human breast cancer cells induces apoptosis and inhibits tumor growth in vitro and in vivo. Breast Cancer Res 10: 1-12.

18. Baik SH, Lee J, Lee YS, Jang JY, Kim CW, et al. (2016) ANT2 shRNA downregulates miR-19a and miR-96 through the PI3K/Akt pathway and suppresses tumor growth in hepatocellular carcinoma cells. Exp Mol Med 48: e222. [Crossref]

19. Jang JY, Kim YG, Nam SJ, Keam B, Kim TM, et al. (2016) Targeting Adenine
Nucleotide Translocase-2 (ANT2) to Overcome Resistance to Epidermal Growth Factor Receptor Tyrosine Kinase Inhibitor in Non-Small Cell Lung Cancer. Mol Cancer Ther 15: 1387-1396.

20. Brower JV, Rodic N, Seki T, Jorgensen M, Fliess N, et al. (2007) Evolutionarily conserved mammalian adenine nucleotide translocase 4 is essential for spermatogenesis. J Biol Chem. 2007;282(40): 29658-29666.

21. Rodic N, Oka M, Hamazaki T, Murawski MR, Jorgensen M, et al. (2005) DNA methylation is required for silencing of Ant 4 , an adenine nucleotide translocase selectively expressed in mouse embryonic stem cells and germ cells. Stem Cells 23 : 1314-1323.

22. Bauer MK, Schubert A, Rocks O, Grimm S (1999) Adenine nucleotide translocase-1, a component of the permeability transition pore, can dominantly induce apoptosis. $J$ Cell Biol 147: 1493-1502. [Crossref]

23. Zamora M, Granell M, Ma mpel T, Vinas O (2004) Adenine nucleotide translocase 3 (ANT3) overexpression induces apoptosis in cultured cells. Febs Lett 563: 155-160.

24. Jang JY, Choi Y, Jeon YK, Kim CW. Suppression of adenine nucleotide translocase-2 by vector-based siRNA in human breast cancer cells induces apoptosis and inhibits tumor growth in vitro and in vivo. Breast Cancer Res 10: R11.

25. Baik SH, Lee J, Lee YS, Jang JY, Kim CW (2016) ANT2 shRNA downregulates miR-19a and miR-96 through the PI3K/Akt pathway and suppresses tumor growth in hepatocellular carcinoma cells. Exp Mol Med 48: e222.

26. Chevrollier A, Loiseau D, Chabi B, Renier G, Douay O, et al. (2005) ANT2 isoform required for cancer cell glycolysis. J Bioenerg Biomembr 37: 307-316. [Crossref]

27. Jang JY, Jeon YK, Kim CW (2010) Degradation of HER2/neu by ANT2 shRNA suppresses migration and invasiveness of breast cancer cells. BMC Cancer 10: 391 [Crossref]

28. Jang JY, Lee YS, Jeon YK, Lee K, Jang JJ, et al. (2013) ANT2 suppression by shRNA restores miR-636 expression, thereby downregulating Ras and inhibiting tumorigenesis of hepatocellular carcinoma. Exp Mol Med 45: 2-15.

29. Vivanco I, Sawyers CL (2002) The phosphatidylinositol 3-Kinase AKT pathway in human cancer. Nat Rev Cancer 2: 489-501. [Crossref]

30. Katso R, Okkenhaug K, Ahmadi K, White S, Timms J (2001) Waterfield MD. Cellular function of phosphoinositide 3-kinases: implications for development, homeostasis, and cancer. Annu Rev Cell Dev Biol 17: 615-675.

31. Samuels Y, Wang Z, Bardelli A, Silliman N, Ptak J, et al. (2004) High frequency of mutations of the PIK3CA gene in human cancers. Science 304: 554. [Crossref]

32. Lee YS, Kim JW, Osborne O, Oh DY, Sasik R, et al. (2014) Increased adipocyte O consumption triggers HIF-1alpha, causing inflammation and insulin resistance in obesity. Cell 157: 1339-1352

33. Park DH, Jung BK, Lee YS, Jang JY, Kim MK, et al. (2015) Evaluation of in vivo antitumor effects of ANT2 shRNA delivered using PEI and ultrasound with microbubbles. Gene Ther 22: 325-332. [Crossref]

Copyright: (C2016 Baik SH. This is an open-access article distributed under the terms of the Creative Commons Attribution License, which permits unrestricted use, distribution, and reproduction in any medium, provided the original author and source are credited. 